Service social

\title{
Comment le nouveau couple vit la recomposition familiale
}

\section{Francine Fortier et Gerry Marino}

Volume 39, numéro 3, 1990

Familles recomposées après divorce

URI : https://id.erudit.org/iderudit/706499ar

DOI : https://doi.org/10.7202/706499ar

Aller au sommaire du numéro

Éditeur(s)

École de service social de l'Université Laval

ISSN

1708-1734 (numérique)

Découvrir la revue

Citer cet article

Fortier, F. \& Marino, G. (1990). Comment le nouveau couple vit la recomposition familiale. Service social, 39(3), 38-49.

https://doi.org/10.7202/706499ar

\section{Résumé de l'article}

Parce qu'elle oblige à une multitude d'ajustements, la recomposition familiale n'est facile pour aucun des acteurs en cause. Toutefois, la survie du nouveau regroupement dépend en bonne partie du couple fondateur. C'est pourquoi il apparaît essentiel de comprendre comment, de son côté, il vit la situation. Dans cette optique, l'article décrit les stress, difficultés et enjeux auxquels sont confrontés les nouveaux époux. 
Francine Fortier, psychologue, pratique privée.

Gerry Marino, psychologue, praticien en thérapie familiale au CLSC Lamater.

\section{Comment le nouveau couple vit la recomposition familiale}

Francine Fortier Gerry Marino

Lorsque les chercheurs ont commencé à s'intéresser à la question du divorce, on percevait celui-ci comme un événement dramatique qui laissait des séquelles graves et permanentes aux enfants. Tandis qu'on se préoccupait surtout d'en cerner les effets, les résultats se contredisaient d'une recherche à l'autre et n'étaient donc que peu concluants. L'étude longitudinale entreprise par Wallerstein et Kelly (1980) finit par mettre en évidence que, si certains enfants restaient affectés, d'autres devenaient des personnes tout aussi équilibrées et compétentes que les jeunes du même âge élevés dans une famille intacte. Cette découverte amena un changement de perspective, si bien qu'aujourd'hui on reconnaît généralement l'existence d'un processus d'adaptation à long terme et on s'intéresse plutôt aux facteurs susceptibles d'influencer l'ajustement (Hetherington et al., 1988; Felner et al., 1988).

Par une étude longitudinale concernant la recomposition familiale, Hetherington et al. (1988) s'attendent à confirmer l'existence d'un processus comparable à celui qu'on a défini relativement au divorce. À la suite du remariage, la famille expérimenterait crises et déséquilibre. Cette période serait suivie d'une restabilisation du système ou, dans les familles qui ne parviennent pas à se rééquilibrer, $d^{\prime}$ une dissolution éventuelle de l'union. Par ailleurs, les tendances observées dans les taux de divorce laissent entendre que la situation n'est pas facile. Selon les calculs de Glick (1988), $55 \%$ à $60 \%$ des remariages aboutiraient à un divorce, comparativement à $49 \%$ des 
premières unions. La moitié de ces ruptures se produiraient dans les cinq ans suivant le remariage, soit deux années plus rapidement que dans le cas d'un premier éclatement.

Si l'on considère que la survie de la nouvelle famille dépend en bonne partie du couple et de sa capacité à surmonter les défis rencontrés, il apparaît fondamental de comprendre comment, de son côté, le couple vit la situation. C'est dans cette optique que nous nous focaliserons ici sur lui. La recomposition sera donc abordée sous I'angle des aspirations du couple, de ses besoins, préoccupations, sentiments et tiraillements. Nous sommes bien conscients que cette fenêtre demeure arbitraire, les époux n'étant pas les seules personnes prises dans la complexité du système. Toutefois, il est d'autant plus important de connaître leur perspective que ce sont souvent ces personnes qui sollicitent notre aide.

Issue d'impressions cliniques et d'un travail de réflexion, la description donnée n'a pas la prétention d'être complète et scientifique. Elle n'est pas non plus axée sur l'élaboration des solutions, une partie de la présente revue se consacrant à cet objectif. Son intention est de clarifier les enjeux de la situation à travers les yeux du couple nouvellement remarié, donnant un aperçu de son état d'esprit lorsqu'il fait appel à nos services. Nous croyons que, compte tenu de l'éclairage attendu des recherches longitudinales, pareille démarche peut être utile. De fait, sans une compréhension des enjeux de la situation et sans une reconnaissance des difficultés vécues par nos clients, nous pouvons difficilement les aider à surmonter leurs frustrations et les guider vers des solutions appropriées.

\section{Le rêve et la réalité}

Comme le soulignent Visher et Visher (1988), la plupart des couples qui s'engagent sur la voie de la recomposition familiale sont peu préparés aux exigences de leur nouvelle vie. Ils s'attendent à recréer une famille comparable à celle de leur enfance ou encore à celle qu'ils ont fondée la première fois. Même lorsqu'ils appréhendent des difficultés, la prégnance de leur amour les porte à regarder l'avenir avec confiance et à accorder une importance minime à l'effet de la cohabitation tout autant qu'aux stress d'adaptation. Les mythes véhiculés le plus souvent sont ceux de l'ajustement et de l'amour instantanés. Globalement, on s'attend à ce que chacun s'adapte rapidement à sa nouvelle famille et, plus particulièrement, à ce que l'enfant et son beau-parent s'aiment automatiquement.

Or, les choses ne peuvent se passer de cette façon. Parce que la juxtaposition d'un projet conjugal sur la myriade des liens antécé- 
dents oblige la redéfinition des relations, des rôles et des territoires, la situation demande une multitude d'ajustements. Ceux-ci sont d'autant plus difficiles à réussir que les enfants et l'autre parent n'abordent pas la recomposition dans le même état d'esprit que les nouveaux conjoints. Ils la voient comme quelque chose qui leur est imposé et se montrent surtout sensibles aux pertes à subir. Comme les intérêts communs sont plutôt rares, l'atteinte d'un équilibre satisfaisant ne peut être qu'un processus de longue haleine. Dans le cours de ce cheminement, les tensions et les frictions sont inévitables, autant entre l'enfant et son beau-parent qu'entre les diverses personnes en cause.

Rêver d'une nouvelle existence est une chose, la vivre en est une autre. Faisant face aux défis de la recomposition, le couple vit sa lune de miel au milieu du chahut. Dans le remue-ménage suscité par la transformation, les difficultés fusent de toutes parts et en même temps. Découvrant une réalité qui fait mentir les mythes, les époux sont aux prises avec diverses ambivalences qui les mènent à $s^{\prime}$ interroger sur le bien-fondé de leur choix.

\section{Les problèmes à l'intérieur de la nouvelle cellule}

Même s'ils sont surtout mus par leurs aspirations conjugales, les nouveaux partenaires ne sont pas sans savoir qu'ils auront à établir un partage entre leur rôle d'époux et leurs responsabilités parentales. Au départ de l'aventure, ils acceptent la contrainte de bon cœur, s'attendant à profiter de leur amour tout en continuant à s'acquitter, comme avant, des devoirs qui leur viennent du passé. Dans les faits, toutefois, construire l'intimité nécessaire à nourrir le couple s'avère souvent un tour de force. $\mathrm{D}^{\prime}$ abord, la présence d'une marmaille déjà bien installée oblige à des corvées répétitives et banales, créant une atmosphère peu propice au romantisme d'une lune de miel. Mais surtout, la multitude d'imprévus suscités par le contexte limite encore davantage les précieux moments qu'on souhaitait réserver à l'union, quand elle ne place pas les époux en situation de conflit.

\section{Les problèmes liés à la transformation des liens}

Parce que la présence des enfants précède la naissance du couple, la simple délimitation du territoire conjugal devient un processus fort compliqué. Comme les premiers n'ont aucune raison de se réjouir du nouveau lien de leur parent, ils manœuvrent tant bien que mal pour défendre leurs intérêts et leurs droits d'ancienneté. II ne leur est évident ni d'accepter que le couple soit là pour rester, ni de 
partager l'amour de leur parent. II n'est donc pas rare qu'ils espèrent secrètement mettre en péril le bonheur des époux, soit pour augmenter leurs chances de voir leurs parents biologiques réunis, soit pour récupérer l'exclusiveté du lien filial. Pour les mêmes raisons, leur premier mouvement en est souvent un de rivalité, voire d'hostilité à l'endroit du nouvel arrivant ou de sa couvée.

$\mathrm{Qu}$ 'elles visent un retour en arrière ou qu'elles servent à défendre d'anciens territoires, les pressions exercées par l'enfant sont susceptibles d'ébranler le couple le plus déterminé. Alors qu'ils ont besoin d'espace et de temps pour consolider leur union, les conjoints se voient contraints de répondre à toutes sortes d'urgences. Aux prises avec un enfant plus provocant, dérangeant ou accaparant qu'à l'accoutumée, forcés d'intervenir dans les innombrables querelles entre les deux fratries, ils se trouvent plus ou moins détournés de leur projet amoureux. Plus la tâche parentale est lourde, et plus les frustrations conjugales s'accumulent. Plusieurs n'arrivent pas à imaginer le bout du tunnel et se prennent à regretter leur vie d'avant.

Inévitablement, l'attitude antagoniste de l'enfant soulève d'importants conflits de loyauté chez le parent, ce qui favorise l'apparition de tensions conjugales et familiales. Quand il voit ses petits résister à l'implantation du lien amoureux et quand il assiste, impuissant, à l'accueil hostile qu'ils réservent à son conjoint, le parent est porté à se sentir déloyal envers eux. $S^{\prime}$ il se sent coupable de leur avoir fait subir une séparation, il peut aller jusqu'à s'interroger sur la légitimité de sa recherche de bonheur ou encore interroger la pertinence de son choix de partenaire. Certains cèdent à la pression et mettent fin à leurs nouveaux engagements, mais la plupart redoublent d'ardeur pour satisfaire les besoins de leur progéniture en même temps que ceux de leur conjoint. Beaucoup rapportent avoir l'impression de courir perpétuellement entre les deux sans réussir à satisfaire ni l'une ni l'autre des parties.

De fait, si le parent n'investit pas assez dans son projet conjugal, la situation ne va pas laisser son partenaire indifférent. Déjà heurté par les manifestations d'hostilité, de plus en plus frustré dans ses aspirations, celui-ci est susceptible de voir l'enfant comme un redoutable adversaire qui manœuvre pour garder tout à lui la personne qu'il chérit. Des sentiments de jalousie peuvent s'ajouter au ressentiment déjà présent. $S^{\prime}$ il tait ces sentiments, la frustration s'accumule, et s'il réclame sa part du gâteau, il contribue à aiguiser les conflits de loyauté éprouvés par l'époux.

Quant à l'enfant, il a surtout besoin qu'on comprenne ses sentiments et qu'on lui laisse du temps pour accepter la situation. Mais avant de constater qu'il a encore sa place, il ne peut que voir le nouveau venu comme un rival et continuer de résister. S'il parvient à 
ébranler la détermination de son parent, il trouve un terrain fertile pour diviser les conjoints et les détourner de leur but, ce qui contribue à attiser les sentiments rivaux de l'étranger.

Bref, dans les premiers temps de la recomposition, les besoins d'une marmaille éprouvée font concurrence aux aspirations conjugales. A priori, la lutte est souvent inégale car, en regard du lien filial, plus solide et plus ancien, l'union récente est encore chambranlante et tend à paraître illicite. Ainsi, c'est généralement au prix d'efforts à satisfaire les besoins de ses protégés que le parent se convainc de la légitimité de son projet. Au cœur de cette compétition, ses conflits de loyauté permettent à ses enfants de ne pas tout perdre, mais ils l'empêchent très souvent d'effectuer un engagement conjugal clair et incontestable. Tant que perdure pareille situation, les fondations de l'édifice restent précaires et le règne de la rivalité persiste.

\section{Les problèmes liés à la transformation du mode de vie}

Amenant sous le même toit deux adultes et des enfants, la nouvelle cohabitation signe le retour à la famille biparentale. De ce fait, elle suscite une transformation des rôles fixés pendant la phase monoparentale. Cependant, l'absence d'une tradition adaptée à la famille recomposée prive les époux de repères fiables. Forcés d'improviser la redistribution des tâches, ils ont tendance à reproduire les schèmes qui ont marqué leur passé.

Quand la transformation est trop brusque ou radicale, elle engendre d'importantes difficultés supplémentaires en ce qu'elle fait subir à l'enfant une nouvelle gamme de pertes. La vie avec un parent unique comporte en effet ses avantages. L'enfant qui vit dans ce cadre bénéficie souvent d'une plus grande considération et est en meilleure position pour soutirer passe-droits et faveurs. Quand la famille redevient régie par deux adultes, l'équilibre des pouvoirs est rompu. Soumis à la perte de certains privilèges ou à celle d'un rôle qui le valorisait, l'enfant est porté à résister davantage, d'autant que les circonstances le portent aussi à croire qu'on veut usurper la place de l'autre parent.

Cette situation est particulièrement susceptible de se produire avec l'introduction d'une belle-mère dans la famille. Depuis des générations, le domaine familial appartient à la femme et l'homme s'y sent moins à l'aise et moins compétent. Cette conjoncture porte la nouvelle recrue à s'approprier une bonne part de la dimension domestique, surtout quand elle se fixe pour mandat d'être une bonne mère pour les enfants. II n'est pas rare non plus qu'elle cherche à donner le ton en ce qui concerne les règles de la maisonnée, la discipline et l'éducation. Soulagé de trouver une relève, le père lui laisse souvent 
toute latitude. Toutefois, dans le contexte de cette redistribution des rôles, sa compagne se trouve bientôt plus lourdement chargée que lui, tandis que ses enfants voient leur mode de vie bouleversé et leurs sentiments de perte exacerbés.

Dans tous les cas où le nouveau conjoint endosse trop vite les fonctions de parent substitut, et malgré la complicité du parent, l'enfant est porté à défendre le statu quo. Comme il n'est pas en mesure de déceler la bonne volonté qui motive habituellement l'attitude des adultes, il cherche plutôt à discréditer ou à boycotter l'étranger. Frustré par le manque de réciprocité, parachuté dans un rôle qui devient vite ingrat, ce dernier finit par se sentir impuissant, incompétent et amer à l'égard de l'enfant qui résiste. Tout cela dégénère parfois en manifestations plus ou moins flagrantes d'hostilité, manifestations qui auront tôt fait d'exacerber à leur tour les conflits de loyauté éprouvés par le parent.

La conjoncture est particulièrement pénible quand le nouveau venu cherche à refaire l'enfant à sa façon ou à imposer une nouvelle loi dans la maisonnée. Quel que soit le bien-fondé de l'intention initiale, l'enfant n'est pas disposé à modifier ses habitudes pour accommoder les exigences d'une personne à qui il n'a pas encore appris à faire confiance et dont le statut lui apparaît illicite. II tend donc à livrer une lutte acharnée, tandis que son beau-parent, impuissant, est tenté d'aiguiser davantage ses armes. Face à l'escalade des conflits qui en résultent, le couple est soumis à un stress intense.

Même quand le nouveau conjoint n'a nullement l'intention de s'improviser parent, son introduction dans la famille donne inévitablement lieu à un choc des cultures. Comme les uns et les autres n'ont pas le même passé familial, les mêmes habitudes et règles de vie, les façons de faire et les valeurs divergent, jusqu'à parfois sembler incompatibles. Tandis que les deux modes de vie s'entrechoquent, il devient de plus en plus impossible pour le beau-parent de rester spectateur. Comment, par exemple, pourrait-elle réussir à se taire quand l'enfant de son partenaire lui impose une corvée quotidienne de ménage, alors qu'elle a patiemment habitué ses propres enfants à ramasser leurs affaires ? Comment pourrait-il tenir sa langue plus d'un mois quand, tous les jours, il est nez à nez avec un enfant de six ans qu'il trouve polisson et mal élevé ? Bref, peu importe les arrangements qu'on a décidés, il est pratiquement impossible d'éliminer les risques de frictions entre l'enfant et le beau-parent, qui ont, somme toute, à s'habituer à vivre sous le même toit sans pour cela bénéficier des atouts de la relation amoureuse. Il est également illusoire d'espérer éviter les conflits au sujet des enfants et la négociation d'un modus vivendi approprié au nouveau regroupement. 
Malheureusement, tant que les mythes dominent dans leur esprit, les époux ont tendance à considérer les différends comme indésirables plutôt qu'inévitables. La conjoncture les porte plutôt à se laisser submerger par divers tiraillements qui les empêchent de relever adéquatement les défis rencontrés.

\section{Le spectre de la méchante belle-mère}

Ces tiraillements sont dus à l'existence d'un autre mythe puissant qui hante habituellement l'inconscient de la nouvelle famille, celui de la méchante belle-mère. Tous et chacun savent en effet que peu de parents iraient jusqu'à troquer le bonheur de leur progéniture contre une recherche égoïste de satisfactions conjugales. Or, tandis que le roman d'amour entre l'enfant et le beau-parent se révèle plus difficile à écrire qu'on ne l'avait imaginé, le spectre de la marâtre pend comme une épée de Damoclès, affectant, d'une manière différente, l'un et I'autre des époux.

Éprouvé par la conjoncture déjà décrite, le beau-parent en vient à ressentir des émotions qui vont à l'encontre des attentes, d'autant que divers autres handicaps relationnels l'empêchent de se lier instantanément. Comprendre et accepter un enfant déjà grandi dans lequel il ne se reconnaît pas et sur lequel il n'a pas de prise ne lui est pas toujours évident. On ne doit pas oublier non plus que, en plus d'être malaisé, le contact est forcé, car il n'a pas fait l'objet d'un choix véritable. Au bout du compte, la présence de l'enfant se révèle donc lourde à porter et difficile à assumer pour une personne qui aspirait plutôt à une vie conjugale paisible et fructueuse. Dans les circonstances, il n'est cependant pas si simple de reconnaître cet état de choses et encore moins de l'exprimer. Tiraillé entre l'obligation d'aimer l'enfant et ses ambivalences, le nouveau conjoint ne sait que faire d'émotions qu'il juge indésirables. À force de répression, celles-ci finissent pourtant par ressortir, créant un choc plus dommageable que l'appropriation de sentiments qui, en définitive, sont compréhensibles et légitimes. En effet, quand il assiste aux inévitables escarmouches qui n'étaient pas prévues au programme, le parent se sent de plus en plus mal à l'aise dans ses engagements récents. Déjà éprouvés par l'attitude de l'enfant, les époux se trouvent à nouveau divisés car le parent a de plus en plus l'impression que sa quête personnelle de bonheur impose à ses protégés la confrontation injustifiée avec un parent non idéal.

Tandis que les époux doivent devenir solidaires dans la négociation d'un nouveau modus vivendi et dans I'acquittement des fonctions parentales, le déroulement des événements tend plutôt à accentuer leurs différences de statut. Bientôt, le beau-parent se trouve en 
position défavorable pour faire valoir ses besoins, ses exigences et ses opinions. De fait, les difficultés qu'il éprouve dans son contact avec l'enfant font surgir le spectre de la méchante belle-mère et contribuent à l'idéalisation des liens biologiques : quand il fait de bonnes choses pour les enfants, il est un bon parent; mais quand il cherche à discuter les règles de la maisonnée, le comportement d'un enfant, la manière de l'éduquer ou le temps qu'on lui accorde, le beau-parent devient l'ogre ou la marâtre. Comme elle n'aide ni le rapport avec l'enfant ni la consolidation de l'alliance entre les époux, cette situation retarde la réorganisation nécessaire de la maisonnée. Pour entamer une négociation plus fructueuse, le couple devra résoudre cet important dilemme, dégageant le beau-parent de la position insoutenable dans laquelle le placent les mythes et développant une vision plus réaliste de la cellule qu'il a fondée.

\section{Les problèmes entre les deux maisonnées}

La vie du nouveau couple est encore compliquée par l'existence d'un second noyau familial pour l'enfant. Contrairement aux ruptures d'époux sans descendance, les anciens partenaires se voient obligés d'entretenir le contact même si l'un d'entre eux se remarie. Or, la collaboration entre toutes ces personnes est rarement facile à installer, même quand tous et chacun savent qu'il y va du confort général et, surtout, du bien-être de l'enfant. Encore ici, les différences de points de vue, d'intérêts en cause et de statuts priment.

Comme le divorce émotionnel n'est pas toujours réglé, des mésententes au sujet des arrangements interfamiliaux sont susceptibles de subsister. La perception des rôles et des responsabilités n'étant pas unanime, divers moyens peuvent être utilisés pour convaincre l'adversaire d'adopter son point de vue ou pour le forcer à s'y soumettre*. Inévitablement reportées dans la nouvelle famille, ces tensions sont accentuées par les circonstances, le parent remarié se trouvant un allié pour poursuivre la bataille, tandis que son ancien partenaire bénéficie de raisons supplémentaires pour l'alimenter.

De fait, anciennes responsabilités et nouveaux engagements se nuisent réciproquement. On n'a qu'à songer à la pension alimentaire, qui est rarement suffisante aux yeux de la mère des enfants et presque toujours trop élevée à ceux de la nouvelle compagne. Même quand les arrangements ont été préalablement négociés à la satisfaction des deux parties, l'intégration d'un nouveau partenaire impose généralement des réajustements importants. Puisque le parent remarié est maintenant lié par de nouveaux engagements, il ne peut afficher ni la 
même souplesse, ni la même disponibilité dans l'acquittement de ses anciennes responsabilités.

Entraîné dans une situation qu'il n'a pas choisie, menacé par la perte de ses prérogatives, l'ancien partenaire est porté à voir la tierce personne comme un ennemi. Fort de ses droits ancestraux, il essaie de l'ignorer et se montre peu compréhensif à l'endroit de ses besoins. Comme il n'a pas d'intérêt personnel à accommoder l'étranger, il fait aussi pression sur son ex-conjoint pour le maintien du statu quo, déplorant son changement d'attitude ou le critiquant. Le climat d'antagonisme est encore amplifié par le beau-parent qui finit souvent par bouillir et par entrer dans l'arène, tandis que son époux se voit à nouveau tiraillé entre ses anciennes et ses nouvelles allégeances.

La lutte risque de s'intensifier quand, par surcroît, l'ancien partenaire se sent menacé dans sa fonction de parent. Comme la famille recomposée expose l'enfant à deux figures parentales du même genre, les circonstances portent à la rivalité. II suffit que le parent concerné se montre soucieux de protéger son exclusivité pendant que son adversaire cherche à se faire reconnaître. La compétition est d'autant plus difficile à éviter que la propension de l'enfant à idéaliser l'absent contribue à l'amorcer.

Par ailleurs, il existe nécessairement des divergences de méthodes et de styles éducatifs entre les deux milieux. Conjoncture et rivalité aidant, chacun est susceptible d'attribuer à l'autre les problèmes qu'il constate chez l'enfant. Or, comme la nouvelle cohabitation soumet ce dernier à des stress d'adaptation, l'apparition de comportements réactionnels est pratiquement inévitable : régressions mineures, troubles psychosomatiques légers, baisse du rendement général ou accentuation de tendances plus ou moins pathologiques. Peu importe leur envergure, dans le contexte d'une lutte préalable pour imposer son point de vue et ses façons de faire, les arguments sont plus souvent dans le camp de l'autre parent qui se croit autorisé par les circonstances.

Même si l'autonomie de chaque milieu semblait acquise avant la recomposition, la situation est propice à raviver d'anciennes luttes, particulièrement quand I'ancien partenaire n'a pas trouvé un lien de remplacement. Comme le mettent en évidence Ahrons et Rodgers (1987), voir son ex-conjoint amoureux d'une autre personne s'avère ingrat pour celui qui est resté célibataire. En martelant le dernier clou dans le cercueil de l'ancienne union, le remariage risque de rouvrir les plaies de cette personne, enclenchant une autre étape dans le processus de sa guérison. C'est donc souvent dans le contexte d'une première recomposition que se joue la dernière manche d'un inévitable combat pour tourner la page sur le passé. Comme c'est par l'intermédiaire de la fonction parentale que les anciens partenaires restent 
réunis, l'enfant, la manière dont on l'éduque, la façon dont on s'acquitte de ses responsabilités à son égard deviennent les enjeux apparents d'une bataille qui témoigne plutôt de la difficulté à laisser aller et à s'autonomiser.

Au cœur de cette lutte, se situe la frontière entre les deux milieux. Pour les nouveaux époux, la démarcation est vitale. L'enjeu dépasse en effet la protection du lien actuel pour englober l'intégrité du nouveau regroupement. Toutefois, pour garantir l'autonomie de leur cellule, ils peuvent avoir à livrer un combat des plus éprouvants. Les résistances s'expriment sous la forme d'ingérences diverses et plus ou moins marquées. Fort de sa légitimité parentale, l'adversaire tente d'imposer ses propres volontés et traditions sur un terrain qui devrait appartenir au nouveau couple. La situation est choquante et ajoute un stress majeur. Comme les époux n'ont pas le pouvoir de transformer l'autre parent, ce n'est qu'à force de frustration et, parfois, de coups échangés, qu'ils finissent par trouver le moyen de se protéger.

Déjà complexes avant la recomposition, les relations entre les deux maisonnées sont susceptibles de se transformer profondément avec l'arrivée d'un tiers partenaire. Alors qu'un climat sain et confortable favorise l'adaptation de l'enfant, on peut rarement s'attendre à ce qu'il s'installe d'emblée. Pour les adultes, qui n'ont pas été entraînés au partage nécessaire des pouvoirs, la situation demande un temps d'apprentissage, sans compter que l'intensité émotionnelle associée à la conjoncture ajoute souvent à la confusion.

\section{Une lutte pour la légitimité}

Essentiellement, le couple fondateur d'une famille recomposée se bat pour établir un sens de légitimité. Nous avons voulu mettre en relief les trois facettes de cette lutte : légitimité de l'union, droits du beau-parent comme membre à part entière de la famille, droit de rester maître chez soi malgré la survivance de l'ancien couple parental. Sans la conviction qu'il a droit à un peu de bonheur, le couple ne survivra pas; sans l'espoir de participer à la définition d'une nouvelle identité familiale, le beau-parent finira par démissionner; et sans la levée $d^{\prime}$ une frontière suffisamment marquée entre les deux maisonnées, l'intégrité du nouveau regroupement sera perpétuellement remise en question. Tant que les époux $n^{\prime}$ ont pas acquis un sens solide de légitimité dans ces trois aires, la famille est soumise à des stress importants. Toutefois, le processus est loin d'être automatique car, $s^{\prime}$ ils ont leur territoire à ériger et à défendre, ils doivent, en même temps, s'accommoder des exigences des autres acteurs. Comme les 
besoins de l'ancien système entrent en compétition avec ceux du nouveau, et puisque l'initiative leur appartient, ils portent d'abord le blâme de la transformation. C'est donc au prix de sentiments de culpabilité et d'ouverture aux autres qu'ils doivent délimiter le champ de leur juridiction et se convaincre de leur compétence et de l'importance de leur projet.

Peuplé d'hésitations et d'ambivalences, ce cheminement n'est pas facile. Il suppose d'abord une multitude de renoncements frustrants. II met aussi en jeu la capacité à transformer son idéal de départ et I'habileté à saisir où réside son véritable pouvoir. Rien n'est plus terrible que de se sentir impuissant et dépossédé. Dès lors, on comprend mieux qu'ils sont nombreux à abandonner avant la fin du parcours. Devant l'échec à faire une famille à la hauteur de leurs aspirations, plusieurs ne parviennent pas à surmonter leurs sentiments de culpabilité et d'impuissance. Ce n'est donc pas en ajoutant au blâme que nous aiderons nos clients à maîtriser les défis rencontrés, mais plutôt en reconnaissant les difficultés liées à la situation et en les guidant dans leur cheminement vers une réappropriation adéquate de leur pouvoir.

À notre avis, la famille recomposée est un lieu de vie valable pour les enfants. Une fois surmontés les principaux obstacles à une bonne adaptation, ils sont en mesure de profiter de différents avantages. Sans négliger les blessures du début, il nous apparaît tout aussi crucial de nous attarder au couple, car de sa survie dépend celle de la nouvelle famille. D'ailleurs, n'est-ce pas dans la mesure où adultes et enfants trouvent leurs places respectives au sein du regroupement que l'on obtient la meilleure garantie de permanence?

\section{Note}

* Pour une discussion approfondie de cette question, surtout liée au genre, voir L. Turgeon (1989).

\section{Références bibliographiques}

Ahrons, C.R. et R.H. Rodgers (1987). Divorced Families : Meeting the Challenge of Divorce and Remariage. New York : W.W. Norton \& Co.

FelNer, R.D., L. TerRe et R.T. Rowlison (1988). " A Life Framework for Understanding Marital Dissolution and Family Reorganisation ", dans WOLCHIK, S.A. et P. KarolY, Children of Divorce : Empirical Perspectives on Adjustment. New York et Londres : Gardner Press. 
Glıck, P.C. (1988). "The Role of Divorce in the Changing Family Structure : Trends and Variations", dans WOLCHIK, S.A. et P. KAROLY, Children of Divorce, op. cit.

Hetherington, E.M., J.D. Arnett et E.A. Hollier (1988). " Adjustment of Parents and Children to Remarriage ", dans Children of Divorce, op. cit.

TURGEON, L. (1989). Le père séparé. Montréal : Les éditions internationales Alain Stanké.

VISHER, E.B. et J.S. VISHER (1988). Old Loyalties, New Ties: Therapeutic Strategies with Stepfamilies. New York : Brunner-Mazel.

Wallerstein, J. et J. Kelly (1980). Surviving the Breakup. New York : Basic Books. 